\title{
Depressive mixed state: Evidence for a new form of depressive state in type I and II bipolar patients
}

\author{
Katia M'Bailara' \\ Donatienne Van den Bulke ${ }^{2}$ \\ Nicolas Demazeau ${ }^{2}$ \\ Jacques Demotes-Mainard ${ }^{3}$ \\ Chantal Henry' \\ 'EA4I39 Laboratoire de psychologie, \\ Université Victor Segalen, Bordeaux \\ Cedex, France; ${ }^{2}$ Centre Hospitalier \\ Charles Perrens, Bordeaux Cedex, \\ France; ${ }^{3}$ INSERM-DRCT, ECRIN, Paris, \\ France
}

Correspondence: Chantal Henry Centre Hospitalier Charles Perrens, Bâtiment Lescure, 121 rue de la Béchade, F-33076 Bordeaux Cedex, France

Tel +33556563450

Fax +33556563547

Email chenry@ch-perrens.fr
Background: A high proportion of unipolar and bipolar type II patients can present a depressive mixed state (DMX). This state is defined by an association of a major depressive episode with at least two specific hypomanic symptoms. This state seems underdiagnosed and this could have treatment implications. The aims of our study were: (i) to investigate the frequency of DMX in type I and II bipolar patients hospitalized for a severe or resistant depressive episode and (ii) to assess the therapeutic response in naturalistic conditions.

Methods: Forty-two consecutive bipolar patients referred by psychiatrists for a severe or resistant depressive episode were assessed using the French version of the Mini International Neuropsychiatric Interview 5.0 (MINI 5.0), which assesses the suicide risk and provides DSM-IV diagnosis. The intensity of mood episodes was evaluated using the MADRS and Bech-Rafaelsen Mania Scale. One group of patients included patients presenting only depressive symptoms (ie, pure major depressive episode (MDE)), and the second group included patients with a major depressive episode and at least two specific hypomanic symptoms (DMX).

Results: Twenty-one patients (50\%) had a pure MDE and 21 patients $(50 \%)$ had a DMX. The treatment leading to recovery was very different in the two groups. Antidepressants were effective (77\%) in MDE patients, whereas antipsychotics were effective (81\%) in DMX. 38\% of patients with a MDE also received a mood stabilizer versus $86 \%$ in the group of DMX. Five MDE patients (24\%) and one DMX patient required electroconvulsive therapy. The suicidal ideations did not differ between the two groups $(\mathrm{p}=0.7)$.

Conclusions: Some mood episodes in bipolar patients (type I and II) are characterised by depressive and hypomanic symptoms but do not meet criteria for mixed episode as defined by DSM-IV. These episodes are often diagnosed as depressive states, but are worsened by antidepressants and often considered as resistant depression. They rapidly respond to antimanic treatment. New categories of mood disorders should take into account this particular mixed state.

Keywords: bipolar depression, mixed state, depressive mixed state, resistant depression

\section{Introduction}

Mixed states are defined by DSM-IV as the co-occurrence of a full manic episode and a full depressive episode. Such states are rather uncommon and most authors consider that manic episodes associated with depressive symptoms are more frequent (McElroy et al 1992, 1995; Bauer et al 1994; Akiskal et al 1998). The DSM-IV (1994) definition of mixed states excludes bipolar II disorder. However, major depressive episodes with only a few submanic symptoms, called depressive mixed state (DMX), have been reported in the literature but not in the DSM-IV (Benazzi 2001a, 2001b, 2003; Benazzi and Akiskal 2001; Perugi et al 2001). Benazzi (2001a, 2001b) studied the number of hypomanic symptoms during major depressive episodes to assess the prevalence of "depressive mixed states" in a cohort of bipolar II and unipolar depressed outpatients. He found that $90 \%$ of patients had at least one hypomanic symptom, and that $28.5 \%$ had three or more. However, these symptoms were more frequent in bipolar (BP) II subjects, with three or more hypomanic symptoms in $48.7 \%$ of subjects. The most prevalent 
symptoms were irritable mood, increased talkativeness, distractibility and racing thoughts. These results were confirmed in a larger collaborative cohort (Benazzi and Akiskal 2001). Agitated depressive mixed state with psychotic features has also been found in BP I patients (Perugi et al 2001). Most authors concluded that the recognition of these affective states is clinically important to protect patients from the potentially harmful indiscriminate use of antidepressants and to provide them with the benefits of anticonvulsants, short-term neuroleptic treatment or electroconvulsive therapy (ECT) (Koukopoulos and Koukopulos 1999).

The aims of this study were: (i) to investigate the frequency of DMX (as defined by an association with a major depressive episode and at least two specific hypomanic symptoms) in a cohort of type I and II bipolar patients hospitalised for a severe or resistant major depressive episode and (ii) to assess the therapeutic response in a naturalistic manner.

\section{Methods}

We recruited 42 bipolar patients consecutively admitted to a specific unit dedicated to mood disorders in Charles Perrens Hospital, Bordeaux (France). All these patients were referred to the hospital by a psychiatrist for a severe or resistant depressive episode. After giving informed consent, patients were interviewed using the French version of the Mini International Neuropsychiatric Interview (M.I.N.I), providing DSM-IV Axis I diagnoses (Sheehan et al 1998). We divided patients into two groups as a function of the presence of hypomanic symptoms. The first group included patients presenting a major depressive state without any hypomanic symptom, the pure major depressive episode (EDM). The second group included patients with a major depressive state associated with at least two specific hypomanic symptoms, defining a depressive mixed state (DMX).

We assessed the suicide risk in the two groups. Patients were considered to be at risk of committing suicide if they answered yes to at least one of the seven questions included in the MINI. This risk is characterized by three levels: mild $=1 ;$ moderate $=2$ and high $=3$. We also recorded any actual suicide attempts. The severity of the mood episode was quantified by the Montgomery and Asberg Depression Rating Scale (MADRS) score (Montgomery and Asberg 1979) and the Bech and Rafaelsen manic scale score (Bech et al 1978) in the group of patients with a DMX. Patients were considered as recovered when they did not meet criteria for a thymic episode and had a score less than 12 on the depression scale and less than 6 on the mania scale. We compared the treatment leading to recovery in the two groups.
Statistical analyses were performed with the SPSS V.10.0 software, using the $\chi 2$-test and the Student's t-test for group comparisons.

\section{Results \\ Sample characteristics}

This study included 30 (71\%) women and 12 men (29\%). The mean age at interview was 46 -years $( \pm 17$; range). Seventy per cent of patients were bipolar type I. Twenty-one patients $(50 \%)$ only met items for major depressive episode, defining the pure MDE group. The same number (21 patients, $50 \%$ ) presented a major depressive episode and hypomanic symptoms, defining DMX.

\section{Clinical characteristics of the two groups (MDE and DMX) (Tablel)}

Patients with DMX had a mean of $4.95( \pm 0.86)$ (range 3-6) hypomanic symptoms. The 2 most frequent specific hypomanic symptoms were: irritability $(73 \%)$, and racing or crowded thoughts (77\%).

The scores on the MADRS depression scale were statistically different between the two groups, with a mean score of $30( \pm 7)$ for the MDE group and $23( \pm 8)$ for the DMX group ( $\mathrm{p}=0.008)$. The mania score in the DMX group was $13( \pm 3)$. Suicidal ideation was present in $17 \mathrm{MDE}$ patients $(80 \%)$ and $15 \mathrm{DMX}$ patients $(72 \%)(\mathrm{p}=0.7)$. There was no difference in the level of suicide risk or the number of real suicide attempts between the two groups (respectively $\mathrm{p}=0.5$ and $\mathrm{p}=1)$.

\section{Response to treatment (Table 2)}

The treatment leading to recovery was very different between the two groups (Table 2). Antidepressants led to recovery in $77 \%$ of MDE patients. Antidepressants were associated with a mood stabilizer in $38 \%$ of cases. Thirteen (62\%) DMX patients were receiving antidepressants upon admission. This treatment was discontinued in all cases except two, in

Table I Clinical characteristics of the two groups

\begin{tabular}{llll}
\hline & MDE & DMX & \\
\hline Mean of hypomanic symptoms & 0 & $4.95( \pm 0.86)$ & \\
MADRS score & $30( \pm 7)$ & $23( \pm 8)$ & $\mathrm{P}=0.008$ \\
$\begin{array}{l}\text { Bech score } \\
\text { Suicidal risk (Yes/no) }\end{array}$ & $17(80 \%)$ & $15(72 \%)$ & $\mathrm{P}=0.7$ \\
$\begin{array}{l}\text { Level of risk (mild }=1, \\
\text { moderate }=2, \text { high }=3)\end{array}$ & 1.86 & 1.62 & $\mathrm{P}=0.5$ \\
$\begin{array}{l}\text { Suicide attempts } \\
\text { (3) }\end{array}$ & $3(15 \%)$ & $4(19 \%)$ & $\mathrm{P}=1$ \\
\hline
\end{tabular}


Table 2 Treatment leading to recovery in the two groups

\begin{tabular}{llll}
\hline & MDE & DMX & \\
\hline Antidepressants & $16(76 \%)$ & $2(9.5 \%)$ & $\mathrm{P}<0.001$ \\
Mood stabilisers & $8(38 \%)$ & $18(86 \%)$ & $\mathrm{P}=0.004$ \\
Antipsychotics & $3(14 \%)$ & $17(81 \%)$ & $\mathrm{P}<0.00 \mathrm{I}$ \\
Benzodiazepines & $3(14 \%)$ & $3(14.3 \%)$ & $\mathrm{P}=1$ \\
ECT & $5(24 \%)$ & $\mathrm{I}(4.8 \%)$ & $\mathrm{P}=0.18$ \\
\hline
\end{tabular}

which the dose was decreased (these two patients have been received antidepressant for many years). In this group, $81 \%$ of patients recovered when given antipsychotic treatment in addition to a mood stabilizer. The antipsychotic was usually discontinued before the patient was discharged.

Five patients (24\%) in the MDE group and one patient in the DMX group needed ECT.

\section{Discussion}

This study shows that there are two distinct "depressive states" in bipolar patients: pure major depressive episode and depressive mixed state as defined by Benazzi (2003). This has very important therapeutic implications because major depressive episodes classically responded to antidepressant medication, whereas depressive mixed states were worsened by antidepressants and improved by mood stabilizers associated with an antipsychotic for a short period. DMX patients may be in-patients with type I or II bipolar disease.

The depression scale scores differed between the two groups. However, the mean was compatible with the diagnosis of depressive episodes in both. Conversely, the mania scale score was not very high because most DMX patients were only mildly agitated. Indeed, this score was closer to those observed in hypomanic episodes rather than in manic episodes strengthening that these patients did not respond to mixed state as defined by DSM-IV. Although some features (particularly the risk of suicide) appeared to be similar in the two groups, the response to treatment differed (recovery under antidepressant vs recovery under antimanic treatment). These two different responses to treatment support the validity of two distinct depressive states in bipolar patients.

This study highlights the fact that DMX patients are worsened by antidepressants, with $62 \%$ of these patients receiving this type of medication before hospitalization. However, hospitalization was required because of an increase in suicidal ideation, agitation or a great inner tension. These findings are of relevance to the controversy of antidepressant and suicidality in some forms of depression in bipolar patients (Dilsaver et al 2005; Mc Elroy et al 2006). DMX state was not due to a switch induced by antidepressants because the patients did not meet the criteria for a manic, hypomanic or mixed state. Moreover, most of these DMX patients were considered by their psychiatrist to show resistant depression with an indication for ECT. However, only one DMX patient required ECT. Akiskal and Mallya (1987), on a case series have reported that patients referred for refractory depressive states had been over-treated with tricyclic antidepressants and had improved with lithium augmentation or with neuroleptics used in low dose.

In a recent study, Bottlender and collegues (2004) have shown that depressed bipolar I patients with mixed features are a high risk for maniform switch during acute treatment. This supports the idea for a different treatment response as a function of the presence of hypomanic symptoms during bipolar depressions.

Our study highlights the problem of the boundaries between the different mood states in bipolar patients and addresses the issue of the real nature of DMX and its links to mixed state, dysphoric mania and hypomanic state (Benazzi and Akiskal 2003). This state is characterized by a mixture of hypomanic and depressive symptoms, and the treatment response is similar to those of mixed states, or manic and hypomanic episodes. However, these patients do not fit the DSM-IV criteria for mixed state, because this state seems closer to a mixture of a hypomanic episode and a depressive one. Unfortunately, we are unable to classify this state satisfactorily using the DSM-IV criteria (Benazzi 2001a, 2001b, 2003; Benazzi and Akiskal 2001).

As the current mood disorder categories are based on the tonality of affects, ie, sadness for MDE, and euphoria and irritability for manic state, it is difficult for a psychiatrist to diagnosis a condition other than depression when a patient complains of sadness, pain, anxiety or suicidal ideation and thus to give him or her a treatment other than an antidepressant. When patients are worsened by this medication, the psychiatrist often considers that the patient displays resistant depression. However two recent studies have shown efficacy of atypical antipsychotics in bipolar depression states (Tohen et al 2003; Calabrese et al 2005). Thus, further research is needed to characterize in which depression bipolar states clinicians have to choose either an antidepressant or an antipsychotic.

\section{Acknowledgments}

This work was supported by the Programme Hospitalier de Recherche Clinique (PHRC: AOM 98/52) and by the Délégation à la Recherche Clinique de l'Assistance Publique des Hôpitaux de Paris. 


\section{References}

Akiskal HS, Hantouche EG, Bourgeois ML, et al. 1998. Gender, temperament, and the clinical picture in dysphoric mixed mania: findings from a French national study (EPIMAN). J Affect Disord, 50:175-86.

Akiskal HS, Mallya G. 1987. Criteria for the "soft" bipolar spectrum: treatment implications. Psychopharmacol Bull, 23:68-73.

American Psychiatric Association. 1994. DSM-IV, diagnostic and statistical manual of mental disorders (IVth ed). Washington DC.

Bauer MS, Whybrow PC, Gyulai L, et al. 1994. Testing definitions of dysphoric mania and hypomania: prevalence, clinical characteristics and inter-episode stability. $J$ Affect Disord, 32:201-11.

Bech P, Rafaelsen OJ, Kramp P, et al. 1978. The mania rating scale: scale construction and inter-observer agreement. Neuropharmacology, 17:430-1.

Benazzi F. 2001a. Major depressive episodes with hypomanic symptoms are common among depressed outpatients. Comprehensive Psychiatry, 42:139-43.

Benazzi F. 2001b. Atypical depression with hypomanic symptoms. J Affect Disord, 65:179-83.

Benazzi F. 2003a. Bipolar II depressive mixed state: finding a useful definition. Comprehensive Psychiatry, 44:21-7.

Benazzi F. 2003b. Depression with racing thoughts. Psychiatry Research, 120:273-82.

Benazzi F, Akiskal HS. 2001. Delineating bipolar II mixed states in the Ravenna-San Diego collaborative study: the relative prevalence and diagnostic significance of hypomanic features during major depressive episodes. J Affect Disord, 67:115-22.

Benazzi F, Akiskal HA. 2003. The dual factor structure of self-rated $M D Q$ hypomania: energized-activity versus irritable-thought racing. $J$ Affect Disord, 73:9-64.
Bottlender R, Sato T, Kleindienst N, et al. 2004. Mixed depressive features predict maniform switch during treatment of depression in bipolar I disorder. J Affect Disord, 78:149-52.

Dilsaver SC, Benazzi F, Rihmer Z, et al. 2005. Gender, Suicidality and bipolar mixed states in adolescent. J Affect Disord, 87:11-6.

Koukopoulos A, Koukopoulos A. 1999. Agitated depression as a mixed state and the problem of melancholia. Bipolarity: beyond classic mania. The psychiatric clinics of North America, 22:564-74.

McElroy SL, Keck PE, Pope HG, et al. 1992. Clinical and research implications of the diagnosis of dysphoric or mixed mania or hypomania. Am J Psychiatry, 149:1633-44.

Mc Elroy SL, Kotwal R, Kaneria R, et al. 2006. Antidepressant and suicidal behaviour in bipolar disorder. Bipolar Disord, 8:569-617.

McElroy SL, Strakowski SM, Keck PE Jr, et al. 1995. Differences and similarities in mixed and pure mania. Comprehensive Psychiatry, 36:187-94.

Montgomery S, Asberg A. 1979. A new depression scale designed to be sensitive to change. Br J Psychiatry, 134:382-9.

Perugi G, Akiskal HS, Micheli C, et al. 2001. Clinical characterization of depressive mixed state in bipolar I patients: Pisa-San Diego collaboration. J Affect Disord, 67:105-14.

Sheehan DV, Lecrubier Y, Sheehan K, et al. 1998. Mini International Neuropsychiatric Interview (M.I.N.I): the development and validation of a structured diagnosis psychiatric interview for DSM-IV and ICD-10. J Clin Psychiatry, 59 (Suppl 20):22-33.

Tohen M, Vieta E, Calabrese J, et al. 2003. Efficacy of olanzapine and olanzapine-fluoxetine combination in the treatment of bipolar I depression. Arch Gen Psychiatry, 60:1079-88. 